\title{
Pseudo-Slant Submanifolds of a Locally Decomposable Riemannian Manifold
}

Mehmet Atçeken, Süleyman Dirik, Ümit Yıldırım

Gaziosmanpasa University, Faculty of Arts and Sciences, Department of Mathematics, 61000 Tokat/TURKEY mehmet.atceken@gop.edu.tr

Amasya University, Faculty of Arts and Sciences, Department of Statistic, 05200, Amasya/TURKEY suleyman.dirik@amasya.edu.tr

Gaziosmanpasa University, Faculty of Arts and Sciences, Department of Mathematics, 61000 Tokat/TURKEY

\section{ABSTRACT}

umit.yildirim@gop.edu.tr

In this paper, we study pseudo-slant submanifolds of a locally decomposable Riemannian manifold. We give necessary and sufficient conditions for distributions which are involued in the definition of pseudo-slant submanifold to be integrable. We search these type submanifolds with parallel canonical structures and we obtain some new results.

\section{Keywords}

Riemannian manifold; Riemannian space form; pseudo-slant submanifold.

2010 AMS Mathematics Subject Classification: 53B25, 53C15, 53C42, 53C55.

\section{Council for Innovative Research}

Peer Review Research Publishing System

Journal: JOURNAL OF ADVANCES IN MATHEMATICS

Vol .11, No.8

www.cirjam.com, editorjam@gmail.com 


\section{INTRODUCTION}

Study of slant submanifolds was initiatedy by B. Y. Chen [3,4], as a generalization of both holomorphich and totally real submanifolds of a Keahler manifold. Slant submanifolds have been studied in different kind structure such as almost contact, neutral Keahler, Lorentzian Sasakian and Sasakian by several geometers.

Semi-slant submanifolds of a Keahler manifold was introduced by N. Papaghich [5], as a naturel generalization of slant submanifolds. After then, bi-slant submanifolds was worked in a almost Hermitian manifold.

Recently, A. Carriazo defined and studied bi-slant submanifolds in an almost Hermitian manifold and gave the some notions of pseudo-slant submanifold in an almost Hermitian manifold[2].

After then, V. A. Khan and M. A. Khan [7], defined and studied the contact versions of pseudo-slant submanifold in a Sasakian manifold.

H. M. Taştan and F. Özdemir studied the pseudo-slant submanifolds in a locally product Riemannian manifold. They obtained a basic inequality involving Ricci curvature and squared mean curvature of a pseudo-slant submanifold of a locally product Rieannian manifold[9].

The purpose of the present paper is to define and study pseudo-slant submanifolds in a locally decomposable Riemannian manifold and work integrability conditions of distributions these submanifolds and type submanifolds with parallel canonical structures. Moreover, an example is used demonstrate that the method presented in this paper is effective.

\section{PRELIMINARIES}

Let $M$ be an $n$-dimensional manifold with a tensor $F$ of type $(1,1)$ such that $F^{2}=I, F \neq \mp I$. In this case $(M, F)$ is said to be an almost product structure $F$. Since $F^{2}=I$, we can set

$$
P=\frac{1}{2}(I+F), \quad Q=\frac{1}{2}(I-F),
$$

then we have

$$
P+Q=I, P^{2}=P, Q^{2}=Q, P Q=Q P=0, \quad F=P-Q .
$$

Thus $P$ and $Q$ define two complementary distributions. We easily see that the eigenvalues of $F$ are 1 and -1 . An eigenvector corresponding to the eigenvalue 1 is in $P$, and an eigenvector corresponding to -1 is in $Q$. Thus if $F$ has eigenvalue 1 of multiplicity $P$ and eigenvalue and eigenvalue -1 of multiplicity $q$, then the dimensions of $P$ is $p$ and that of $Q$ is $q$.

Conversely, there exit in $M$ two globally complementary distributions $P$ and $Q$ of dimensions $p$ and $q$, respectively $(p+q=n)$, then we can define an almost product structure $F$ on $M$ by $F=P-Q$.

If an almost product manifold $(M, F)$ admits a Riemannian metric $g$ such that

$$
g(X, Y)=g(F X, F Y)
$$

for any vector fields $X, Y$ on $M$, then $M$ is called an almost product Riemannian manifold.

Moreover, If the almost Riemannian product structure $F$ is parallel that is, $\left(\nabla_{X} F\right)=0$, then $(M, F, g)$ is called locally decomposable Riemannian manifold[10].

If $M_{1}\left(c_{1}\right)$ is a real space form with sectional curvature $c_{1}$ and $M_{2}\left(c_{2}\right)$ is a real space form with sectional curvature $c_{2}$, then the Riemannian curvature tensor $R$ of locally decomposable Riemannian manifold $M_{1}\left(c_{1}\right) \times M_{2}\left(c_{2}\right)$ is given by

$$
\begin{aligned}
R(X, Y) Z= & \frac{1}{4}\left(c_{1}+c_{2}\right)\{g(Y, Z) X-g(X, Z) Y+g(F Y, Z) F X \\
& -g(F X, Z) F Y\}+\frac{1}{4}\left(c_{1}-c_{2}\right)\{g(F Y, Z) X \\
& -g(F X, Z) Y+g(Y, Z) F X-g(X, Z) F Y\}
\end{aligned}
$$


for any vector fields $X, Y$ and $Z$ on $\tilde{M}$.

In this section, we will recall the definitions an some notations used throughout this paper. For an arbitrary submanifold $\tilde{M}$ of a Riemannian manifold $M$ the Gauss and Weingarten formulas are, respectively, given by

$$
\nabla_{X} Y=\tilde{\nabla}_{X} Y+h(X, Y)
$$

and

$$
\nabla_{X} Y=-A_{V} X+\nabla_{X}^{\perp} V
$$

for any vector fields $X, Y$ tangent to $\tilde{M}$ and $V$ normal to $\tilde{M}$, where $\nabla$ and $\tilde{\nabla}$ denote the Riemannian connections on $M$ and $\tilde{M}$, respectively. On the oyther hand, $h: \Gamma(T \tilde{M}) \times \Gamma(T \tilde{M}) \rightarrow \Gamma\left(T^{\perp} \tilde{M}\right)$ is the second fundamental form and $A_{V}: \Gamma(T \tilde{M}) \rightarrow \Gamma(T \tilde{M})$ is also the shape operator of $\tilde{M}$ in $M$, where $\Gamma(T \tilde{M})$ denotes the differentiable vector fields set on $\tilde{M} . \nabla^{\perp}$ is the normal connection on the normal bundle $\Gamma\left(T^{\perp} \tilde{M}\right)$. On the other hand, $A_{V}$ and $h$ are related by the formula

$$
g\left(A_{V} X, Y\right)=g(h(X, Y), V),
$$

for any $X, Y \in \Gamma(T \tilde{M})$ and $V \in \Gamma\left(T^{\perp} \tilde{M}\right)$.

If we denote the Riemannian curvature tensor of the connection $\nabla$ by $R$ then Gauss and Codazzi equations are, respectively, given by formulas

and

$$
\begin{aligned}
R(X, Y) Z= & \tilde{R}(X, Y) Z+A_{h(X, Z)} Y-A_{h(Y, Z)} X \\
& +\left(\nabla_{X} h\right)(Y, Z)-\left(\nabla_{Y} h\right)(X, Z)
\end{aligned}
$$

$$
(R(X, Y) Z)^{\perp}=\left(\nabla_{X} h\right)(Y, Z)-\left(\nabla_{Y} h\right)(X, Z),
$$

for any $X, Y, Z \in \Gamma(T \tilde{M})$, where $(R(X, Y) Z)^{\perp}$ denotes normal part of $R(X, Y) Z$. If $(R(X, Y) Z)^{\perp}=0$, then the submanifold $\tilde{M}$ is said to be curvature invariant.

Let $\tilde{M}$ be a submanifold a locally decomposable Riemannian manifold $(M, F, g)$. Then we can write

$$
F X=f X+\omega X
$$

for any $X \in \Gamma(T \tilde{M})$, where $f X$ and $\omega X$ denote the tangent and normal components of $F X$, respectively. In same way, we have

$$
F V=B V+C V
$$

for any $V \in \Gamma\left(T^{\perp} \tilde{M}\right)$, where $B V$ and $C V$ are also the tangent and normal components of $F V$, respectively. By using (8) and (9) and the properties of $F$, we obtain

$$
f^{2}+B \omega=I, \quad \omega f+C \omega=0
$$

and

$$
f B+B C=0, \quad \omega B+C^{2}=I .
$$

If $f=0$ (res. $\omega=0$ ), $\tilde{M}$ is said to be an anti-invariant (resp. an invariant) submanifold. Moreover, if $f \neq 0$ and $\omega \neq 0$, then $\tilde{M}$ is called semi-invariant submanifold. 


\section{PSEUDO-SLANT SUBMANIFOLDS}

Let $\tilde{M}$ be a submanifold of a locally decomposable Riemannian manifold $(M, F, g)$. A distribution $D$ on $\tilde{M}$ is said to be a slant if for $X \in D_{p}$ the angle $\theta$ between $F X$ and $D_{p}$ is constant, that is, it is independent of $p \in M$ and $X \in D_{p}$. The constant angle $\theta$ is called the slant angle of the distribution. So a submanifold $\tilde{M}$ of $M$ is said to be a slant submanifold if the tangent bundle $T \tilde{M}$ of $\tilde{M}$ is slant[8].

Thus invariant and anti invariant submanifolds are special cases of slant submanifolds.

Definition 3.1. Let $\tilde{M}$ be a submanifold of a locally decomposable Riemannian manifold $(M, F, g)$. $\tilde{M}$ is said to be pseudo-slant of $M$ if there exit two distributions $D^{\perp}$ and $D^{\theta}$ on $\tilde{M}$ such that

i) $T \tilde{M}$ has the orthogonal direct decomposition $T \tilde{M}=D^{\perp} \oplus D^{\theta}$

ii) The distribution $D^{\perp}$ is an anti-invariant, that is, $F\left(D^{\perp}\right) \subseteq T^{\perp} \tilde{M}$,

iii) The distribution $D^{\theta}$ is a slant distribution with slant angle $\theta$.

Next, we denote $p$ and $q$ the dimensions of $D^{\perp}$ and $D^{\theta}$, respectively, then we have the following classifications;

i) If $p=0$, then $\tilde{M}$ is an anti-invariant submanifold.

ii) If $q=0$ and $\theta=0$, then $\tilde{M}$ is invariant submanifold.

iii) If $q=0$ and $\theta \neq\left\{0, \frac{\pi}{2}\right\}$, then $\tilde{M}$ is a proper slant submanifold.

iv) If $p q \neq 0$ and $\theta=0$, then $\tilde{M}$ is semi-invariant submanifold.

v) If $p q \neq 0$ and $\theta \neq\left\{0, \frac{\pi}{2}\right\}$, then $\tilde{M}$ is a pseudo-slant submanifold[7].

Now, let $\tilde{M}$ be a pseudo-slant submanifold of a locally decomposable Riemannian manifold $(M, F, g)$ and we denote the orthogonal complementary of $F\left(D^{\perp}\right)$ and $F\left(D^{\theta}\right)$ in $T^{\perp} \tilde{M}$ by $v$, then we have direct sum

$$
T^{\perp} \tilde{M}=F\left(D^{\perp}\right) \oplus F\left(D^{\theta}\right) \oplus v .
$$

We note that $F\left(D^{\perp}\right)$ and $F\left(D^{\theta}\right)$ are mutually orthogonal distributions in normal bundle because $D^{\perp}$ and $D^{\theta}$ are orthogonal distributions.

The following theorem characterize pseudo-slant submanifolds of in a locally product Riemannian manifolds.

Theorem 3.1. Let $\tilde{M}$ be a submanifold of a locally Riemannian product manifold $(M, F, g)$. Then $\tilde{M}$ is a slant submanifold if and only if there exists a constant $\lambda \in(0,1)$ such that $f^{2}=\lambda . I$. In this case, if $\theta$ is the slant angle of $\tilde{M}$, then it is satisfies $\lambda=\cos ^{2} \theta$.

Theorem 3.1. Let $\tilde{M}$ be a submanifold of a locally decomposable Riemannian manifold $(M, F, g)$. Then $\tilde{M}$ is a pseudo-slant submanifold if and only if there exists a constant $\lambda \in(0,1)$

and a distribution $D^{\theta}$ on $\tilde{M}$ such that

i) $D^{\theta}=\{X \in T \tilde{M} \mid B \omega X=\lambda X\}$

ii) For $X \in T \tilde{M}$ orthogonal to $D^{\theta}, B \omega X=X$. Furthermore, if $\theta$ is slant angle, it is satisfies $\lambda=\sin ^{2} \theta$. 
Proof. We suppose that $\tilde{M}$ is a pseudo-slant submanifold. Then for $X \in D^{\theta}$, from Theorem 3.1 and (10), we obtain $B \omega X=\sin ^{2} \theta X$, i.e., i) is satisfied. For $X \in \Gamma(T \tilde{M})$ orthogonal to $D^{\theta}, f^{2} X=0$, also it implies that $B \omega X=X$. So ii) is also satisfied.

Conversely, the conditions i) and ii) are satisfied. We put $\lambda=\sin ^{2} \theta$, from (1) and (10), for $X \in D$, we obtain $f^{2} X=X \cos ^{2} \theta$ which proves $D$ is a slant distribution. We denote the orthogonal distribution of $D$ in $\tilde{M}$ by $D^{\prime}$, for $X \in D^{\prime}$, , from ii), we can easily see $B \omega X=X$, that is, $f^{2} X=0$. This tell us $D^{\prime}$ is an anti-invariant distribution.

Now, we will give an example to show that we work on space is nonempty.

Example 3.1. We consider the Euclidean space $\square^{6}=\square^{3} \mathrm{X} \square^{3}$ with usual Riemannian metric and Riemannian product structure $F$.

Let $\tilde{M}$ be submanifold of $\square^{6}$ defined by

$$
\begin{gathered}
x(u, v, s, t)=(\sqrt{13} t, 2 u+v+3 s+t, u+2 v-3 s-2 t, \\
u+v-3 t,-u-3 s-3 t, v-3 s) .
\end{gathered}
$$

Then the tangent bundle of $\tilde{M}$ are spanned by the vectors

$$
\begin{aligned}
& e_{1}=2 \frac{\partial}{\partial x_{2}}+\frac{\partial}{\partial x_{3}}+\frac{\partial}{\partial x_{4}}-\frac{\partial}{\partial x_{5}}, \quad e_{2}=\frac{\partial}{\partial x_{2}}+2 \frac{\partial}{\partial x_{3}}+\frac{\partial}{\partial x_{4}}+\frac{\partial}{\partial x_{6}} \\
& e_{3}=3 \frac{\partial}{\partial x_{2}}-3 \frac{\partial}{\partial x_{3}}-3 \frac{\partial}{\partial x_{5}}-3 \frac{\partial}{\partial x_{6}}, \quad e_{4}=\sqrt{13} \frac{\partial}{\partial x_{1}}+\frac{\partial}{\partial x_{2}}-2 \frac{\partial}{\partial x_{3}}-3 \frac{\partial}{\partial x_{4}}-3 \frac{\partial}{\partial x_{5}}
\end{aligned}
$$

where $\left(x_{1}, x_{2}, x_{3}, x_{4}, x_{5}, x_{6}\right)$ are usual coordinates of $\square^{6}$ and $\left\{\frac{\partial}{\partial x_{i}}\right\}, 1 \leq i \leq 6$ are standart basis vector fields of $E^{6}$. Then we can easily to see that

$$
\begin{array}{ll}
F e_{1}=2 \frac{\partial}{\partial x_{2}}+\frac{\partial}{\partial x_{3}}-\frac{\partial}{\partial x_{4}}+\frac{\partial}{\partial x_{5}}, & F e_{2}=\frac{\partial}{\partial x_{2}}+2 \frac{\partial}{\partial x_{3}}-\frac{\partial}{\partial x_{4}}-\frac{\partial}{\partial x_{6}}, \\
F e_{3}=3 \frac{\partial}{\partial x_{2}}-3 \frac{\partial}{\partial x_{3}}+3 \frac{\partial}{\partial x_{5}}+3 \frac{\partial}{\partial x_{6}}, & F e_{4}=\sqrt{13} \frac{\partial}{\partial x_{1}}+\frac{\partial}{\partial x_{2}}-2 \frac{\partial}{\partial x_{3}}+3 \frac{\partial}{\partial x_{4}}+3 \frac{\partial}{\partial x_{5}} .
\end{array}
$$

According to the product structure $F$ and the usual metric tensor $g$ of $\square^{6}$, we obtain

$$
\begin{aligned}
& g\left(F e_{3}, e_{1}\right)=g\left(F e_{3}, e_{2}\right)=g\left(F e_{3}, e_{3}\right)=g\left(F e_{3}, e_{4}\right)=0, \\
& g\left(F e_{4}, e_{1}\right)=g\left(F e_{4}, e_{2}\right)=g\left(F e_{4}, e_{3}\right)=g\left(F e_{4}, e_{4}\right)=0
\end{aligned}
$$

and

$$
\cos \theta=\frac{g\left(F e_{1}, e_{1}\right)}{\left\|F e_{1}\right\|\left\|e_{1}\right\|}=\frac{g\left(F e_{1}, e_{2}\right)}{\left\|F e_{1}\right\|\left\|e_{2}\right\|}=\frac{g\left(F e_{2}, e_{2}\right)}{\left\|F e_{2}\right\|\left\|e_{2}\right\|}=\frac{3}{7} .
$$

Thus the slant distribution $D^{\theta}=\operatorname{Span}\left\{e_{1}, e_{2}\right\}$ and anti-invariant distribution $D^{\perp}=\operatorname{Span}\left\{e_{3}, e_{4}\right\}$, that is, $\tilde{M}$ is a 4dimensional pseudo-slant submanifold of $\square^{6}$ with usual almost Riemannian product structure $F$ and metric tensor $g$. Let $\tilde{M}$ be a pseudo-slant submanifold of a locally decomposable Riemannian manifold $(M, F, g)$. Then we have

$$
\begin{aligned}
\nabla_{X} F Y & =F \nabla_{X} Y \\
\nabla_{X} f Y+\nabla_{X} \omega Y & =F \tilde{\nabla}_{X} Y+F h(X, Y) \\
h(X, f Y)+\tilde{\nabla}_{X} f Y-A_{\omega Y} X+\nabla_{X}^{\perp} \omega Y & =f \tilde{\nabla}_{X} Y+\omega \tilde{\nabla}_{X} Y+B h(X, Y)+C h(X, Y)
\end{aligned}
$$


for any $X, Y \in \Gamma(T \tilde{M})$. Corresponding the tangent and normal components of (13), we get

$$
\left(\tilde{\nabla}_{X} f\right) Y=A_{\omega Y} X+B h(X, Y)
$$

and

$$
\left(\nabla_{X} \omega\right) Y=-h(X, f Y)+C h(X, Y) .
$$

In the same way, for any $X \in \Gamma(T \tilde{M})$ and $V \in \Gamma\left(T^{\perp} \tilde{M}\right)$, we obtain

$$
\begin{gathered}
\nabla_{X} F V=F \nabla_{X} V \\
\nabla_{X} B V+\nabla_{X} C V=F\left(-A_{V} X\right)+F \nabla_{X}^{\perp} V \\
h(X, B V)+\tilde{\nabla}_{X} B V-A_{C V} X+\nabla_{X}^{\perp} C V=-f A_{V} X-\omega A_{V} X+B \nabla^{\perp} V+C \nabla^{\perp}{ }_{X} V,
\end{gathered}
$$

which implies that

$$
\left(\nabla_{X} B\right) V=A_{C V} X-f A_{V} X
$$

and

$$
\left(\nabla_{X} C\right) V=-\omega A_{V} X-h(X, B V) .
$$

On the other hand, for any $Z, W \in \Gamma\left(D^{\perp}\right)$, from (14), we have

$$
-A_{F W} Z=f \nabla_{W} Z+B h(Z, W),
$$

that is,

$$
F[Z, W]=A_{F Z} W-A_{F W} Z
$$

and

$$
\begin{aligned}
g\left(A_{F W} Z-A_{F Z} W, U\right) & =g(h(Z, U), F W)-g(h(W, U), F Z) \\
& =g\left(\nabla_{U} Z, F W\right)-g\left(\nabla_{U} W, F Z\right) \\
& =-g(h(W, U), F Z)+g\left(\nabla_{U} F Z, W\right) \\
& =-g\left(A_{F Z} W, U\right)-g\left(A_{F Z} W, U\right) \\
& =-2 g\left(A_{F Z} W, U\right)
\end{aligned}
$$

which proves

$$
A_{F W} Z=-A_{F Z} W
$$

for all $U \in \Gamma(T \tilde{M})$. From (18) and (19), we conclude that

$$
f[Z, W]=2 A_{F Z} W .
$$

Thus we have the following theorem.

Theorem 3.3. Let $\tilde{M}$ be a pseudo-slant submanifold of a locally decomposable Riemannian manifold $(M, F, g)$. The orthogonal distribution $D^{\perp}$ is integrable if and only if

$$
A_{F D^{\perp}} D^{\perp}=0 .
$$

Theorem 3.4. Let $\tilde{M}$ be a pseudo-slant submanifold of a locally decomposable Riemannian manifold $(M, F, g)$. The slant distribution $D^{\theta}$ is integrable if and only if the second fundamental form $h$ of $\tilde{M}$ satisfies the condition

$$
h(X, f Y)-h(f X, Y) \in \Gamma\left(v \oplus \omega\left(D^{\theta}\right)\right)
$$

for $X, Y \in \Gamma\left(D^{\theta}\right)$. 
Proof. For $X, Y \in \Gamma\left(D^{\theta}\right)$ and $Z \in \Gamma\left(D^{\perp}\right)$, we have

$$
\begin{aligned}
g([X, Y], Z)= & g\left(\nabla_{X} Y, Z\right)-g\left(\nabla_{Y} X, Z\right) \\
& =g\left(\nabla_{Y} Z, X\right)-g\left(\nabla_{X} Z, Y\right) \\
& =g\left(\nabla_{Y} F Z, F X\right)-g\left(\nabla_{X} F Z, F Y\right) \\
& =g\left(\nabla_{Y} \omega Z, f X\right)+g\left(\nabla_{Y} \omega Z, \omega X\right) \\
& -g\left(\nabla_{X} \omega Z, f Y\right)-g\left(\nabla_{X} \omega Z, \omega Y\right) \\
& =-g\left(A_{\omega Z} Y, f X\right)+g\left(A_{\omega Z} X, f Y\right) \\
& +g\left(\left(\nabla_{Y} \omega\right) Z+\omega\left(\nabla_{Y} Z\right), \omega X\right) \\
& -g\left(\left(\nabla_{X} \omega\right) Z+\omega\left(\nabla_{X} Z\right), \omega Y\right) .
\end{aligned}
$$

Taking into account of $v$ and $\omega D^{\theta}$ being mutually orthogonal and (15), we arrive

$$
\begin{aligned}
g([X, Y], Z)= & g(h(X, f Y), \omega Z)-g(h(Y, f X), \omega Z) \\
& +\sin ^{2} \theta g\left(\nabla_{Y} Z, X\right)-\sin ^{2} \theta g\left(\nabla_{X} Z, Y\right) \\
& =g(h(X, f Y)-h(Y, f X), \omega Z) \\
& +\sin ^{2} \theta g\left(\nabla_{X} Y, Z\right)-\sin ^{2} \theta g\left(\nabla_{Y} X, Z\right) \\
& =g(h(X, f Y)-h(Y, f X), \omega Z)+\sin ^{2} \theta g([X, Y], Z),
\end{aligned}
$$

that is,

$$
\cos ^{2} \theta g([X, Y], Z)=g(h(X, f Y)-h(f X, Y), \omega Z),
$$

which proves our assertion.

Theorem 3.5. Let $\tilde{M}$ be a pseudo-slant submanifold of a locally decomposable Riemannian manifold $(M, F, g)$. $\tilde{M}$ is a mixed geodesic submanifold if and only if

$$
A_{\omega B V} Z+A_{\omega Z} B V \in \Gamma\left(D^{\perp}\right)
$$

for $Z \in \Gamma\left(D^{\perp}\right)$ and $V \in \Gamma\left(T^{\perp} \tilde{M}\right)$.

Proof. For any $X \in \Gamma\left(D^{\theta}\right)$ and $Z \in \Gamma\left(D^{\perp}\right)$, we have

$$
\begin{aligned}
g\left(A_{V} X, Z\right) & =g\left(\nabla_{X} Z, V\right)=g\left(\nabla_{X} F Z, F V\right) \\
& =g\left(\nabla_{X} \omega Z, B V\right)+g\left(\nabla_{X} \omega Z, C V\right) \\
& =g\left(\nabla_{X} \omega Z, B V\right)+g\left(\left(\nabla_{X} \omega\right) Z+\omega \nabla_{X} Z, C V\right) .
\end{aligned}
$$

Making use of $C$ being symmetric and (17), we obtain

$$
\begin{aligned}
g\left(A_{V} Z, X\right) & =-g\left(A_{\omega Z} B V, X\right)+g(C h(X, Z), C V) \\
& =g\left(A_{C^{2} V} Z-A_{\omega Z} B V, X\right) \\
& =g\left(A_{V} Z-A_{\omega B V} Z-A_{\omega Z} B V, X\right)
\end{aligned}
$$

which proves our assertion. 
In a pseudo-slant submanifold, slant and anti-invariant distributions are totally geodesic in submanifold, pseudoslant submanifold is called pseudo-slant product.

Theorem 3.6. Let $\tilde{M}$ be a pseudo-slant submanifold of a locally decomposable Riemannian manifold $(M, F, g)$. $\tilde{M}$ is a pseudo-slant product if and only if the shape operatory $A$ of $\tilde{M}$ satisfies

$$
A_{F\left(D^{\perp}\right)} f\left(D^{\theta}\right)=0 \text {. }
$$

Proof. By using (3), (4) and (15), we have

$$
\begin{aligned}
g\left(\nabla_{Z} U, X\right) & =g\left(\nabla_{Z} F U, F X\right) \\
& =g\left(\nabla_{Z} \omega U, f X\right)+g\left(\nabla_{Z} \omega U, \omega X\right) \\
& =-g\left(A_{\omega U} Z, f X\right)+g\left(\left(\nabla_{Z} \omega\right) U+\omega\left(\nabla_{Z} U\right), \omega X\right) \\
& =-g(h(Z, f X), \omega U)+g\left(\omega \nabla_{Z} U, \omega X\right)
\end{aligned}
$$

for any $U, Z \in \Gamma\left(D^{\perp}\right)$ and $X \in \Gamma\left(D^{\theta}\right)$. This implies that

$$
\cos ^{2} \theta g\left(\nabla_{Z} U, X\right)=-g(h(Z, f X), \omega U)=-g\left(A_{\omega U} f X, Z\right) .
$$

Furthermore, by a direct calculation, we reach

$$
\begin{aligned}
g\left(\nabla_{X} Z, Y\right) & =g\left(\nabla_{X} F Z, F Y\right) \\
& =g\left(\nabla_{X} \omega Z, f Y\right)+g\left(\nabla_{X} \omega Z, \omega Y\right) \\
& =-g\left(A_{\omega Z} X, f Y\right)+g\left(\left(\nabla_{X} \omega\right) Z+\omega\left(\nabla_{X} Z\right), \omega Y\right) \\
& =-g(h(X, f Y), \omega Z)+g\left(\omega \nabla_{X} Z, \omega Y\right)
\end{aligned}
$$

for any $X, Y \in \Gamma\left(D^{\theta}\right)$ and $Z \in \Gamma\left(D^{\perp}\right)$. So we have

$$
\cos ^{2} \theta g\left(\nabla_{X} Z, Y\right)=-g\left(A_{\omega Z} f Y, X\right) .
$$

Combining (24) and (25), we conclude that $\tilde{M}$ is a pseudo-slant product if and only if (23) is satisfied.

Theorem 3.7. Let $\tilde{M}$ be a pseudo-slant submanifold of a locally decomposable Riemannian manifold $(M, F, g)$. The tensor field $\omega$ is parallel if and only if shape operator $A_{V}$ satisfies

$$
A_{V} Y=\sec ^{2} \theta A_{C V} f Y
$$

for any $Y \in \Gamma(T \tilde{M})$ and $V \in \Gamma\left(T^{\perp} \tilde{M}\right)$.

Proof. If $\omega$ is parallel, from (15), we have

$$
C h(X, Y)-h(X, f Y)=0,
$$

for any $X, Y \in \Gamma(T \tilde{M})$. This implies

$$
C h(X, f Y)-\cos ^{2} \theta h(X, Y)=0 .
$$

Thus we have

$$
g(C h(X, f Y), V)-\cos ^{2} \theta g(h(X, Y), V)=0
$$

for any $V \in \Gamma\left(T^{\perp} \tilde{M}\right)$. This is equivalent to

$$
A_{V} Y=\sec ^{2} \theta A_{C V} f Y .
$$


The converse is obvious.

For pseudo-slant submanifold $\tilde{M}$ in $M, \tilde{M}$ is called $D^{\perp}-$ geodesic if $h(X, Y)=0$ for any $X, Y \in \Gamma\left(D^{\perp}\right)$.

Theorem 3.8. Let $\tilde{M}$ be a pseudo-slant submanifold of a locally decomposable Riemannian manifold $(M, F, g)$. If the tensor field $f$ is parallel on $\tilde{M}$, then $\tilde{M}$ is a $D^{\perp}-$ geodesic submanifold.

Proof. If $f$ is parallel, then from (14), we have

$A_{\omega X} Y+B h(X, Y)=0$

for any $X, Y \in \Gamma\left(D^{\perp}\right)$. Taking into account of $h$-being symmetric and (19), we conclude $A_{\omega X} Y=B h(X, Y)=0$ and $f \nabla_{X} Y=0$ i.e., $\tilde{\nabla}_{X} Y \in \Gamma\left(D^{\perp}\right)$. Thus we have $g\left(F \nabla_{X} Y, V\right)=g\left(\nabla_{X} Y, F V\right)=g(h(X, Y), C V)=0$, for any $V \in v$. This implies that $C h(X, Y)=0$. So $\tilde{M}$ is $D^{\perp}-$ geodesic submanifold.

Theorem 3.9. Let $\tilde{M}$ be a pseudo-slant submanifold of a locally decomposable Riemannian manifold $(M, F, g)$. If the tensor field $C$ is parallel, then $\tilde{M}$ is $D^{\perp}-$ geodesic submanifold.

Proof. Since $C$ is parallel, from (17), we reach

$$
\omega A_{V} X+h(X, B V)=0
$$

for $X \in \Gamma(T \tilde{M})$ and $V \in \Gamma\left(T^{\perp} \tilde{M}\right)$. Here, taking $V=F Y=\omega Y$ for $Y \in \Gamma\left(D^{\perp}\right)$, we have $\omega A_{\omega Y} X+h(X, B \omega Y)=0$. Making use of (19), we arrive at

$$
h(X, B \omega Y)+h(B \omega X, Y)=0,
$$

$X \in \Gamma\left(D^{\perp}\right)$.

On the other hand, because of $B \omega Y=Y-f^{2} Y=Y$, we get $h(X, Y)=0$. This proves our assertion. By using (15) and (16), we get

$$
g\left(\left(\nabla_{X} B\right) V, Y\right)=g\left(\left(\nabla_{X} \omega\right) Y, V\right),
$$

for $X, Y \in \Gamma(T \tilde{M})$ and $V \in \Gamma\left(T^{\perp} \tilde{M}\right)$.

Theorem 3.10. Let $\tilde{M}$ be a pseudo-slant submanifold of a locally decomposable Riemannian manifold $(M, F, g)$. If the tensor field $B$ is parallel, then linear map

$$
C^{2}: V \rightarrow V
$$

has either $h$ - eigenvector with $\cos ^{2} \theta$ eigenvalue or $\tilde{M}$ is totally geodesic submanifold in $\tilde{M}$.

Proof. Since $B$ is parallel, by using (16), we have

$$
A_{C V} X=f A_{V} X
$$

$X \in \Gamma(T \tilde{M})$ and $V \in \Gamma\left(T^{\perp} \tilde{M}\right)$. Substituting (27) into $V=F Y=\omega Y$ for $Y \in \Gamma\left(D^{\perp}\right)$, we get $f A_{\omega Y} X=0$, that is, $A_{\omega Y} X \in \Gamma\left(D^{\perp}\right)$. Thus $g(h(X, f Z), \omega Y)=0$, which implies $h(X, f Z) \in \Gamma(v)$, for $X, Z \in \Gamma(T \tilde{M})$. Taking into (27), we conclude

$$
C^{2} h(X, Z)=C h(X, f Z)=h\left(X, f^{2} Z\right)=\cos ^{2} \theta h(X, Z) .
$$

This tells us that linear map $C^{2}$ has either eigenvector $h$ with eigenvalue $\cos ^{2} \theta$ or it is totally geodesic submanifold.

Theorem 3.11. Let $\tilde{M}$ be a pseudo-slant submanifold of a locally decomposable Riemannian manifold $(M, F, g)$. If the tensor field $C$ is parallel, then $\tilde{M}$ is a pseudo-slant minimal submanifold. 
Proof. Since $C$ is parallel, from (17), we have

$$
h(X, B H)+\omega A_{H} X=0,
$$

for $X \in \Gamma(T \tilde{M})$, where $H$ denotes the mean curvature tensor of $\tilde{M}$ in $M$. Thus (28) implies

$$
g\left(\omega A_{H} X, H\right)+g(h(X, B H), H)=2 g(h(X, B H), H)=0 .
$$

Here we suppose that $H \neq 0$ and $B H=0$. Then

$$
\omega A_{C H} X+h(X, B C H)=0,
$$

from (11), we mean $\omega A_{C H} X=0$, which from

$$
g\left(A_{C H} X, Y\right)=g(h(X, Y), C H)=0 .
$$

This proves $C H=0$. The proof is complete.

\section{PSEUDO-SLANT SUBMANIFOLD IN LOCALLY DECOMPOSABLE RIEMANNIAN SPACE FORMS}

In this section, we have researched pseudo-slant submanifolds in product Riemannian space forms.

Theorem 4. 1. Let $\tilde{M}$ be a pseudo-slant submanifold of locally decomposable Riemannian space form $M_{1}\left(c_{1}\right) \times M_{2}\left(c_{2}\right)$. If $\tilde{M}$ is a curvature-invariant pseudo-slant submanifold, then $\tilde{M}$ is a proper slant submanifold.

Proof. We suppose that $\tilde{M}$ is curvature-invariant pseudo-slant submanifold of a $M_{1}\left(c_{1}\right) \times M_{2}\left(c_{2}\right)$. From (2) and (6), we have

$$
\left(\nabla_{X} h\right)(Y, Z)-\left(\nabla_{Y} h\right)(X, Z)=\frac{1}{4}\left(c_{1}+c_{2}\right) g(F Y, Z) \omega X+\frac{1}{4}\left(c_{1}-c_{2}\right) g(Y, Z) \omega X=0,
$$

for any $X \in \Gamma\left(D^{\perp}\right)$ and $Y, Z \in \Gamma\left(D^{\theta}\right)$. This implies that

$$
\left\{\left(c_{1}-c_{2}\right) g(Y, Z)+\left(c_{1}+c_{2}\right) g(F Y, Z)\right\} \omega X=0
$$

and

$$
\left\{\left(c_{1}-c_{2}\right) g(F Y, Z)+\left(c_{1}+c_{2}\right) g(Y, Z)\right\} \omega X=0 .
$$

From the solutions of (29) and (30), we conclude the $g(Y, Z) \omega X=0$. This tell us $M$ is a proper slant submanifold.

Now, let $\left\{e_{1}, e_{2}, \ldots, e_{p}, e_{p+1}, e_{p+2}, \ldots, e_{p+q}\right\}$ be a orthonormal basis of $\Gamma(T \tilde{M})$ such that $\left\{e_{1}, e_{2}, \ldots, e_{p}\right\}$ are basis vectors of $\Gamma\left(D^{\theta}\right)$ and $\left\{e_{p+1}, e_{p+2}, \ldots, e_{p+q}\right\}$ are basis vectors $\Gamma\left(D^{\perp}\right)$. We denote the Riemannian curvature and Ricci tensors of $\tilde{M}$ by $\tilde{R}$ and $S$, respectively, by using (2), we have

$$
\begin{aligned}
\tilde{R}(X, Y) Z= & \frac{1}{4}\left(c_{1}+c_{2}\right)\{g(Y, Z) X-g(X, Z) Y+g(F Y, Z) F X \\
& -g(F X, Z) F Y\}+\frac{1}{4}\left(c_{1}-c_{2}\right)\{g(F Y, Z) X \\
& -g(F X, Z) Y+g(Y, Z) F X-g(X, Z) F Y\} \\
& +A_{h(Y, Z)} X-A_{h(X, Z)} Y+\left(\nabla_{Y} h\right)(X, Z)-\left(\nabla_{X} h\right)(Y, Z),
\end{aligned}
$$

and 


$$
\begin{aligned}
S(X, Y)= & \frac{1}{4}\left(c_{1}+c_{2}\right)\left\{\left(p+q-1-\cos ^{2} \theta\right) g(X, Y)+\operatorname{tr}(f) g(f X, Y)\right\} \\
& +\frac{1}{4}\left(c_{1}-c_{2}\right)\{(p+q-2) g(f X, Y)+\operatorname{tr}(f) g(X, Y)\} \\
& +(p+q) g(h(X, Y), H)-\sum_{i, j=1}^{p+q} g\left(h\left(e_{i}, X\right), h\left(e_{j}, Y\right)\right) .
\end{aligned}
$$

Also, scalar curvature $\sigma$ of $\tilde{M}$ is given by

$$
\begin{aligned}
\sigma= & \frac{1}{4}\left(c_{1}+c_{2}\right)\left\{\left(p+q-1-\cos ^{2} \theta\right)(p+q)+\operatorname{tr}^{2}(f)\right\} \\
& +\frac{1}{4}\left(c_{1}-c_{2}\right)\{(p+q-2) \operatorname{tr}(f)+(p+q) \operatorname{tr}(f)\} \\
& +(\mathrm{p}+\mathrm{q})^{2}\|H\|^{2}-\|h\|^{2} .
\end{aligned}
$$

From (23) and (31), we have following Theorem.

Theorem 4. 2. Let $\tilde{M}$ be a pseudo-slant submanifold of a locally decomposable Riemannian space form $M=M_{1}\left(c_{1}\right) \times M_{2}\left(c_{2}\right)$. If $\tilde{M}$ is a totally geodesic submanifold, then $\tilde{M}=\tilde{M}_{1}\left(c_{1}\right) \times \tilde{M}_{2}\left(c_{2}\right)$, where $\tilde{M}_{1}\left(c_{1}\right)$ is a real space form of constant curvature $c_{1}$ and $\tilde{M}_{2}\left(c_{2}\right)$ is a real space form of constant curvature $c_{1}$.

Theorem 4. 3. Let $\tilde{M}$ be a $(p+q)$-dimensional pseudo-slant minimal submanifold of a $2 m$-dimensional locally decomposable Riemannian space form $M=M_{1}(c) \times M_{2}(c)$. Then $\tilde{M}$ is a totally geodesic submanifold if and only if $\tilde{M}$ satisfies one of the following conditions;

i) $\tilde{M}$ is a Riemannian product manifold of two $M^{p}(c)$ and $M^{q}(c)$.

ii) $S=\frac{1}{2} c\left\{\left(p+q-1-\cos ^{2} \theta\right) g(.,)+.\operatorname{tr}(f) g(f .,).\right\}$,

iii) $\sigma=\frac{1}{2} c\left\{\left(p+q-1-\cos ^{2} \theta\right)(p+q)+t r^{2}(f)\right\}$.

\section{REFERENCES}

[1] Atçeken, M. and Hui, S.K. 2009. Slant and pseudo-slant submanifolds in $(L C S)_{n}$ - manifolds. Czechoslovak Math. J. $63,177-190$.

[2] Carriazo, A. 2002. New developments in slant submanifolds theory, Narasa Publishing Hause New Delhi. India.

[3] Chen, B.Y. 1990. Geometry of slant submanifolds. Katholieke Universiteit Leuven, Leuven.

[4] Chen, B.Y. 1990. Slant immersions. Bull. Austral. Math. Soc. 41(1), 135-147.

[5] Papaghuic, N. 1994. Semi-slant submanifolds of a Kaehlarian manifold, An. St. Univ. Al. I. Cuza. Univ. Iasi. 40 55-61.

[6] De, U. C and Sarkar A. 2011. On pseudo-slant submanifolds of trans Sasakian manifolds. Proceedings of th Estonian A.S.60,1-11.2011.doi:10.3176\ proc.2011.1.01.

[7] Khan,V. A. and Khan,M. A. 2007. Pseudo-slant submanifolds of a Sasakian manifold, Indian J. prue appl. Math. $38,31-42$.

[8] Lotta, A. 1996. Slant submanifolds in contact geometry, Bulletin Mathematical Society Roumanie. 39,183-198.

[9] Tastan, H. M and Özdemir, F. 2015. The geometry of hemi-slant submanifolds of a locally product Riemannian manifold, Turk. J. Math.(39)(2015). doi:10.3906 \mat1407-18.

[10] Yano, Y. and Kon, M. 1984. Structures on manifolds. Ser. Prue Math. World Scientific. 